\title{
PERIPHERAL LOCAL SPECTRUM PRESERVERS AND MAPS INCREASING THE LOCAL SPECTRAL RADIUS
}

\author{
ABDellatif Bourhim, TARIK JARI AND JAVAD MASHREGHI
}

Abstract. We address two long standing problems in the context of local spectral radius preservers. First, we completely describe the form of maps preserving the peripheral local spectrum of product or triple product of operators. Second, we establish the automatic continuity of linear maps increasing the local spectral radius of operators at a fixed nonzero vector.

Mathematics subject classification (2010): Primary 47B49; Secondary 47A10, 47A11.

Keywords and phrases: Linear preservers, spectrally bounded map, local spectrum, local spectral radius, the single-valued extension property.

\section{REFERENCES}

[1] P. AiEnA, Fredholm and local spectral theory, with applications to multipliers, Kluwer, Dordrecht, 2004.

[2] B. Aupetit, A primer on spectral theory, Springer-Verlag, New York, 1991.

[3] R. Bhatia, P. ŠEMrl AND A. Sourour, Maps on matrices that preserve the spectral radius distance, Studia Math., 134, No. 2 (1999) 99-110.

[4] A. Bourhim AND J. MASHREghi, A survey on preservers of spectra and local spectra, CRM Proceedings and Lecture Notes: Invariant subspaces of the shift operator, American Mathematical Society, Providence, RI, 2015, to appear.

[5] A. BOURHIM AND J. MASHREGHI, Maps preserving the local spectrum of triple product of operators, Linear and Multilinear Algebra, to appear.

[6] A. Bourhim And J. MAShreghi, Maps preserving the local spectrum of product of operators, Glasgow Math. J., to appear.

[7] A. BOURHIM AND J. MASHREGHI, Local spectral radius preservers, Integral equations and Operator Theory, 76, No. 1, (2013) 95-104.

[8] A. Bourhim, Surjective linear maps preserving local spectra, Linear Algebra and its Applications, 432, No. 1, (2010) 383-393.

[9] A. Bourhim AND V. G. Miller, Linear maps on $M_{n}(\mathbb{C})$ preserving the local spectral radius, Studia Math., 188, No. 1, (2008) 67-75.

[10] A. BOURHIM AND T. RANSFORD, Additive maps preserving local spectrum, Integral equations and Operator Theory, 55 (2006) 377-385.

[11] J. BRAČIČ AND V. MÜLlER, Local spectrum and local spectral radius of an operator at a fixed vector, Studia Math., 194, No. 2, (2009) 155-162.

[12] M. BREŠAR AND P. ŠEMRL, Linear maps preserving the spectral radius, J. Funct. Anal., 142, No. 2, (1996) 360-368.

[13] J. T. ChAn, C. K. Li AND N. S. SZE, Mappings preserving spectra of products of matrices, Proc. Amer. Math. Soc., 135 (2007), 977-986.

[14] C. CostaRA, Local spectrum linear preservers at non-fixed vectors, Linear Algebra and its Applications, 457, No. 15, (2014) 154-161.

[15] C. CostaRA, Surjective maps on matrices preserving the local spectral radius distance, Linear and Multilinear Algebra, 62, No. 7, (2014) 988-994.

[16] C. COSTARA, Linear maps preserving operators of local spectral radius zero, Integral equations and Operator Theory, 73, No. 1, (2012) 7-16. 
[17] C. COSTARA, Automatic continuity for linear surjective mappings decreasing the local spectral radius at some fixed vector, Arch. Math., 95, No. 6, (2010) 567-573.

[18] J. L. CUI AND J. C. Hou, Maps leaving functional values of operator products invariant, Linear Algebra and its Applications, 428 (2008) 1649-1663.

[19] M. ECH-ChERIF El KetTANi AND H. BenbouZiane, Additive maps preserving operators of inner local spectral radius zero, Rendiconti del Circolo Matematico di Palermo (2), 63, No. 2, (2014) 311316.

[20] A. FoŠner And P. ŠEmrl, Spectrally bounded linear maps on $\mathscr{B}(X)$, Canad. Math. Bull., 47, No. 3, (2004) 369-372.

[21] M. GonZÁLeZ And M. MbeKhtA, Linear maps on $M_{n}(\mathbb{C})$ preserving the local spectrum, Linear Algebra and its Applications, 427, No. 2-3, (2007) 176-182.

[22] J. C. Hou, Q. H. DI, Maps preserving numerical range of operator products, Proc. Amer. Math. Soc., 134 (2006) 1435-1446.

[23] L. MolnáR, Some characterizations of the automorphisms of $B(H)$ and $C(X)$, Proc. Amer. Math. Soc., 130 (2002), 111-120.

[24] V. MÜLLER, Spectral theory of linear operators and spectral systems in Banach algebras. Operator Theory: Advances and Applications, 139. Birkhäuser Verlag, Basel, 2003.

[25] A. A. Jafarian And A. R. Sourour, Spectrum-preserving linear maps, J. Funct. Anal. 66 (1986), $255-261$.

[26] T. JARI, Nonlinear maps preserving the inner local spectral radius, Rendiconti del Circolo Matematico di Palermo (2) (To appear).

[27] K. B. Laursen, M. M. Neumann, An introduction to local spectral theory, London Mathematical Society Monograph, New Series 20, 2000.

[28] C. K. LI, P. ŠEMRL, N. S. SZE, Maps preserving the nilpotency of products of operators, Linear Algebra and its Applications, 424 (2007) 222-239.

[29] P. ŠEMRL, Spectrally bounded linear maps on B(H), Quart. J. Math. Oxford, 49 (1998) 87-92.

[30] A. R. Sourour, Invertibility preserving linear maps on $\mathscr{L}(X)$, Trans. Amer. Math. Soc., 348, No. 1, (1996) 13-30.

[31] M. OMLADIČC AND P. ŠEMRL, Additive mappings preserving operators of rank one, Linear Algebra and its Applications, 182 (1993), 239-256.

[32] M. WANG, L. FANG AND G. JI, Linear maps preserving idempotency of products or triple Jordan products of operators, Linear Algebra and its Applications, 429 (2008) 181-189. 